\title{
Periodic solutions and Whitham equations for the
} AB system

\author{
A M Kamchatnov† and M V Pavlovł \\ † Institute of Spectroscopy, Russian Academy of Sciences, Troitsk, Moscow obl., 142092, Russia \\ and Troitsk Institute of Innovational and Thermonuclear Investigations, Troitsk, Moscow obl., \\ 142092, Russia \\ $\ddagger$ L D Landau Institute of Theoretical Physics, Russian Academy of Sciences, Moscow, 117334, \\ Russia
}

Received 1 July 1994, in final form 19 January 1995

\begin{abstract}
Effective formulae for the single-phase periodic solution of the AB system are obtained. The solution depends on a complete set of four complex parameters (Riemann invariants). The derivation of Whitham equations which describe a slow evolution of the modulated periodic solution is presented. The general theory is illustrated by application to the problem of soliton creation on the front of a sharp pulse.
\end{abstract}

\section{Introduction}

As is well known, physical systems exist in which the linear waves are unstable because the frequencies of the corresponding dispersion relation consist of two complex conjugate values in some region of the control parameter $[1,2]$. Such a linear wave will grow untill the nonlinear effects take place. Taking them into account, we obtain the so-called AB system of evolution equations, which has a universal nature and can be applied to very different physical situations [1,2]. The $A B$ system belongs to the family of integrable equations whose soliton solutions can be studied by the inverse scattering transform method. Some applications, however, require knowledge not only of the soliton solutions but of the periodic solutions as well. For example, as was shown in [3], the self-modulation of waves described by the nonlinear Schrödinger (NLS) equation can be investigated by the Whitham averaging method $[4,5]$. In this case a small initial disturbance in the unstable system transforms into an expanding non-uniform region which can be described as a modulated nonlinear periodic wave. The evolution of such a region is governed by the Whitham equations which can be obtained by averaging the evolution equations over small-range oscillations.

In this paper we shall find the periodic solution of the $A B$ system in the effective form by the method suggested in [6] for the NLS and DNLS equations, and applied later to some other equations in $[7,8]$. The Whitham equations for the $\mathrm{AB}$ system will be obtained by means of a convenient modification of the averaging method [7-10]. The theory thus developed provides an effective approach to non-stationary and non-uniform problems described by the $\mathrm{AB}$ system. As an example of such an application, we shall consider the problem of the creation of solitons on the front of a sharp pulse. This theory is analogous to the Gurevich-Pitaevskii approach [11] to so-called nondissipative shock waves, but in this case Whitham characteristic speeds are complex (as in the unstable NLS case $[3,7]$ ), which makes the theory much more complicated. 


\section{Periodic solution}

We take the AB system in its canonical form [1]

$$
Q_{\xi \tau}=Q S \quad S_{\xi}=-\frac{1}{2}\left(|Q|^{2}\right)_{\tau}
$$

where $\xi$ and $\tau$ are semi-characteristic coordinates, $Q$ and $S$ are the wave amplitudes obeying the normalization condition

$$
\left|Q_{\tau}\right|^{2}+S^{2}=1
$$

The inverse scattering transform method is based on the possibility of presenting equations (1) as a compatibility condition of two linear systems:

$$
\begin{array}{ll}
\partial \psi_{1} / \partial \xi=F \psi_{1}+G \psi_{2} & \partial \psi_{1} / \partial \tau=A \psi_{1}+B \psi_{2} \\
\partial \psi_{2} / \partial \xi=H \psi_{1}-F \psi_{2} & \partial \psi_{2} / \partial \tau=C \psi_{1}-A \psi_{2} .
\end{array}
$$

Requiring $\partial^{2} \psi_{1,2} / \partial \xi \partial \tau=\partial^{2} \psi_{1,2} / \partial \tau \partial \xi$, we obtain the equations

$$
\begin{aligned}
& \frac{\partial F}{\partial \tau}-\frac{\partial A}{\partial \xi}+C G-B H=0 \\
& \frac{\partial G}{\partial \tau}-\frac{\partial B}{\partial \xi}+2(B F-A G)=0 \\
& \frac{\partial H}{\partial \tau}-\frac{\partial C}{\partial \xi}+2(A H-C F)=0
\end{aligned}
$$

which coincide with (1) if one sets

$$
\begin{array}{lc}
F=-\mathrm{i} \lambda & G=Q / 2 \quad H=-Q^{*} / 2 \\
A=-\frac{1}{4 \mathrm{i} \lambda} S & B=\frac{1}{4 \mathrm{i} \lambda} Q_{\tau} \quad C=\frac{1}{4 \mathrm{i} \lambda} Q_{\tau}^{*}
\end{array}
$$

where $\lambda$ is an arbitrary spectral parameter.

The system (3) has two basic solutions, $\left(\psi_{1}, \psi_{2}\right)$ and $\left(\phi_{1}, \phi_{2}\right)$, which can be used to build a vector with the spherical components

$$
f=-\frac{\mathrm{i}}{2}\left(\psi_{1} \phi_{2}+\psi_{2} \phi_{1}\right) \quad g=\psi_{1} \phi_{1} \quad h=-\psi_{2} \phi_{2}
$$

satisfying the following linear systems:

$$
\begin{array}{ll}
\partial f / \partial \xi=-\mathrm{i} H g+\mathrm{i} G h & \partial f / \partial \tau=-\mathrm{i} C g+\mathrm{i} B h \\
\partial g / \partial \xi=2 \mathrm{i} G f+2 F g & \partial g / \partial \tau=2 \mathrm{i} B f+2 A g \\
\partial h / \partial \xi=-2 \mathrm{i} H f-2 F h & \partial h / \partial \tau=-2 \mathrm{i} C f-2 A h .
\end{array}
$$

During the evolution the length of the vector with components (7) is preserved, that is the quantity

$$
f^{2}-g h=P(\lambda)
$$

is independent of $\xi$ and $\tau$. The periodic solution is distinguished by the condition that $P=P(\lambda)$ be a polynomial in $\lambda$. The single-phase solution corresponds, as we shall see, to the fourth-degree polynomial

$$
P(\lambda)=\prod_{i=1}^{4}\left(\lambda-\lambda_{i}\right)=\lambda^{4}-s_{1} \lambda^{3}+s_{2} \lambda^{2}-s_{3} \lambda+s_{4}
$$


where the $\lambda_{i}$ are its zeros. It is easy to find that the systems (8) are satisfied if we take

$$
f=\lambda^{2}-f_{1} \lambda+a S \quad g=\frac{Q}{2} \lambda+\mathrm{i} a Q_{\tau} \quad h=-\frac{Q^{*}}{2} \lambda+\mathrm{i} a Q_{\tau}^{*}
$$

where $f_{1}$ and $a$ are some constants. Their values can be obtained by substituting equation (11) in (9) and comparing the coefficients of $\lambda^{3}$ and $\lambda^{0}$ :

$$
f_{1}=\frac{s_{1}}{2}=\frac{1}{2} \sum_{i=1}^{4} \lambda_{i} \quad a^{2}=s_{4}=\prod_{i=1}^{4} \lambda_{i} \quad a=-\sqrt{s_{4}} .
$$

(See below about the choice of the sign before the root.)

Now it is convenient to introduce the point of the auxiliary spectrum

$$
\mu=-2 \mathrm{i} a \frac{Q_{\tau}}{Q}
$$

so that

$$
g=\frac{Q}{2}(\lambda-\mu) \quad h=-\frac{Q^{*}}{2}\left(\lambda-\mu^{*}\right) .
$$

If we substitute equation (14) in (8) and put $\lambda=\mu$, then we shall obtain the evolution equations for $\mu$ :

$$
\frac{\partial \mu}{\partial \xi}=-2 \mathrm{i} f(\mu)=-2 \mathrm{i} \sqrt{P(\mu)} \quad \frac{\partial \mu}{\partial \tau}=\frac{1}{4 a} \frac{\partial \mu}{\partial \xi} .
$$

Thus, $\mu$ depends only on the phase

$$
W=\xi+\frac{1}{4 a} \tau \quad \frac{\mathrm{d} \mu}{\mathrm{d} W}=-2 \mathrm{i} \sqrt{P(\mu)} .
$$

The condition that the phase velocity of the nonlinear wave lies between two values of group velocities of the initial physical system (these group velocities enter into the definitions of the variables $\xi$ and $\tau$ ) requires $a \leqslant 0$ (see [1]), what was used in (12) and will be assumed to hold in what follows. In particular, such a choice leads in the limit of infinite wavelength to stable solitons.

One can find from (8), (11), (13) and (14) that

$$
Q=\exp \left(-\frac{i f_{1}}{2 a} \tau\right) \tilde{Q}
$$

provided $\tilde{Q}$ satisfies the equation

$$
\frac{\mathrm{d} \tilde{Q}}{\mathrm{~d} W}=2 \mathrm{i}\left(\mu-\frac{s_{\mathrm{l}}}{2}\right) \tilde{Q}
$$

The equations thus derived form the basis of the 'finite-gap' method for obtaining the periodic solutions. From now on we shall follow the method of [6].

In the case of the $A B$ system, as in many others, the operators corresponding to (3), (5), (6) are not self-adjoint and, hence, their eigenvalues, which coincide with the zeros $\lambda_{i}, i=1,2,3,4$, of the polynomial $P(\lambda)$, may be complex. Therefore the variable $\mu$ moves along some curve which defines the contour of integration when one calculates $\mu(W)$ according to (16). It seems natural to determine this contour explicitly for $\mu$ by means of introducing some coordinate parameter $\nu$ along it. The dependence $\mu(\nu)$ must be such that the constraint (9) is satisfied identically. After substitution of (11) and (14), the constraint (9) takes the form

$$
\left(\lambda^{2}-f(\lambda+a S)^{2}+\frac{1}{4}|Q|^{2}(\lambda-\mu)\left(\lambda-\mu^{*}\right)=P(\lambda)\right.
$$


As in [6], we choose the variable

$$
\nu=\left|Q^{2}\right| / 4
$$

as a parameter along the contour of $\mu$. Comparing coefficients of the $\lambda^{k}$ on both sides of (19), we obtain the equations

$$
\begin{aligned}
& 2 f_{1}=s_{1} \quad f_{1}^{2}+2 a S+v=s_{2} \\
& 2 f_{1} a S+v\left(\mu+\mu^{*}\right)=s_{3} \quad(a S)^{2}+v \mu \mu^{*}=s_{4}
\end{aligned}
$$

which coincide with the corresponding system in the case of the NLS equation [6]. Its solution has the form

$$
f_{1}=\frac{s}{2} \quad a s=\frac{s^{2}}{16}+\frac{p-v}{2} \quad \mu=\frac{s}{2}-\frac{q+\mathrm{i} \sqrt{-K(\nu)}}{2 v}
$$

where

$$
R(v)=v^{3}-2 p v^{2}+\left(p^{2}-4 r\right) v+q^{2}
$$

is the cubic resolvent of the polynomial $P(\lambda)$ whose zeros $\nu_{j}, j=1,2,3$, are related to the zeros $\lambda_{i}, i=1,2,3,4$, by simple symmetrical formulae:

$$
\begin{aligned}
& v_{1}=-\frac{1}{4}\left(\lambda_{1}-\lambda_{2}+\lambda_{3}-\lambda_{4}\right)^{2} \\
& v_{2}=-\frac{1}{4}\left(\lambda_{1}-\lambda_{2}-\lambda_{3}+\lambda_{4}\right)^{2} \\
& v_{3}=-\frac{1}{4}\left(\lambda_{1}+\lambda_{2}-\lambda_{3}-\lambda_{4}\right)^{2} .
\end{aligned}
$$

The parameters $s, p, q, r$ are connected with the coefficients of $P(\lambda)$ according to

$$
\begin{aligned}
& s=s_{1} \quad p=s_{2}-\frac{3}{8} s_{1}^{2} \quad q=\frac{1}{2} s_{1}\left(s_{2}-\frac{1}{4} s_{1}^{2}\right)-s_{3} \\
& r=s_{4}+\frac{1}{16} s_{1}^{2}\left(s_{2}-\frac{3}{16} s_{1}^{2}\right)-\frac{1}{4} s_{1} s_{3} .
\end{aligned}
$$

As in [6], the zeros $\lambda_{i}$ consist of the two complex conjugate pairs $\lambda_{1}, \lambda_{3}$ and $\lambda_{2}, \lambda_{4}$

$\lambda_{1}=\alpha+\mathrm{i} \gamma \quad \lambda_{2}=\beta+\mathrm{i} \delta \quad \lambda_{3}=\alpha-\mathrm{i} \gamma \quad \lambda_{4}=\beta-\mathrm{i} \delta$

so that equations (24) yield

$$
v_{1}=-(\alpha-\beta)^{2} \quad \nu_{2}=(\gamma-\delta)^{2} \quad v_{3}=(\gamma+\delta)^{2} .
$$

The positive (according to definition (20)) variable $\nu$ oscillates in the interval $\nu_{2} \leqslant v \leqslant \nu_{3}$, so that $\mu(v)$ is a cubic curve going around the zeros $\lambda_{1}, \lambda_{3}=\lambda_{1}^{*}$ (see [6]). The dependence of $v$ on the phase $W$ is governed by the equation

$$
\frac{d v}{d(2 W)}=\sqrt{-R(v)}
$$

derived in [6]. Integration of this equation yields the simple expression for dependence of $|Q|^{2}$ on $W$ :

$$
\begin{aligned}
|Q|^{2} & =4 v \\
& =4\left[\nu_{3}+\left(\nu_{2}-v_{3}\right) \operatorname{sn}^{2}\left(\sqrt{\nu_{3}-\nu_{1}} W, m\right)\right] \\
& =4(\gamma+\delta)^{2}-16 \gamma \delta \operatorname{sn}^{2}\left\{\left[(\gamma+\delta)^{2}+(\alpha-\beta)^{2}\right]^{1 / 2} W, m\right\}
\end{aligned}
$$

where we use the more convenient parameter

$$
m=\frac{\nu_{3}-\nu_{2}}{\nu_{3}-\nu_{1}}=\frac{4 \gamma \delta}{(\gamma+\delta)^{2}+(\alpha-\beta)^{2}}
$$


instead of the usual $k=\sqrt{m}$, and the initial condition corresponds to the maximum value of the intensity $|Q|^{2}=4(\gamma+\delta)^{2}$ at $W=0$. If we know $v$, we can easily find $S$ from the second expression in (22).

It is convenient to express the variable $Q$ in terms of Weierstrass elliptic functions as was done in [6] for the solution of the NLS equation. Equation (29) corresponds to

$$
v=\frac{2}{3} p-4 \wp\left(2 W+\omega^{\prime}\right)
$$

where $\omega^{\prime}$ is a half-period of the $\wp$-function. Substitution of (31) into (22) gives the expression for $\mu$ as a function of $W$ :

$$
\mu=\frac{s}{4}+\frac{q}{8} \frac{1}{\wp\left(2 W+\omega^{\prime}\right)-p / 6}-\frac{\mathrm{i}}{2} \frac{1}{v} \frac{\mathrm{d} \nu}{\mathrm{d}(2 W)} .
$$

Integration of (18) with the help of (32) yields

$$
\tilde{Q}=2 \sqrt{v} \exp \left[\frac{\mathrm{i} s}{2} W+\frac{\mathrm{i} q}{8} \int_{0}^{W} \frac{\mathrm{d}(2 W)}{\wp\left(2 W+\omega^{\prime}\right)-p / 6}\right]
$$

where the integral can be calculated as in [6]. The final expression for $Q$ has the form

$$
\begin{gathered}
Q(\tau, \xi)=2(\gamma+\delta) \exp \left[-\frac{\mathrm{i}(\alpha+\beta) \tau}{2 \sqrt{\left(\alpha^{2}+\gamma^{2}\right)\left(\beta^{2}+\delta^{2}\right)}}-\mathrm{i}(\alpha+\beta) W\right. \\
\left.-2 W\left(\zeta(\chi)-\frac{\eta \chi}{\omega}+\frac{\mathrm{i}\left(\gamma^{2}-\delta^{2}\right)}{2|\alpha-\beta|}\right)\right] \frac{\theta_{4}(0) \theta_{3}[(2 W+\chi) / 2 \omega]}{\theta_{4}(W / \omega) \theta_{3}(\chi / 2 \omega)} \\
W=\xi-\frac{\tau}{4 \sqrt{\left(\alpha^{2}+\gamma^{2}\right)\left(\beta^{2}+\delta^{2}\right)}}
\end{gathered}
$$

where the $\theta_{k}$ are the Jacobi theta-functions, $\zeta(\chi)$ is the Weierstrass $\zeta$-function, the parameter $\chi$ is determined by

$\sqrt{(\gamma+\delta)^{2}+(\alpha-\beta)^{2}} \chi=2 \mathrm{i} \int_{0}^{\sin \varphi} \frac{\mathrm{d} z}{\sqrt{\left(1-z^{2}\right)\left(1-m^{\prime} z^{2}\right)}}=2 \mathrm{i} F\left(\varphi, m^{\prime}\right)$

$m^{\prime}=1-m$, and the angle $\varphi$ has simple geometrical meaning

$$
\sin \varphi=\frac{|\alpha-\beta|}{\sqrt{(\alpha-\beta)^{2}+(\gamma-\delta)^{2}}} .
$$

Let us consider some limiting cases of this general periodic solution. Let $\beta=\alpha$, i.e. all $\lambda_{i}$ lie on one vertical line. Then, as in [6], we find

$$
Q(\xi, \tau)=2(\gamma+\delta) \exp \left[-\frac{\mathrm{i} \alpha \tau}{2 \sqrt{\left(\alpha^{2}+\gamma^{2}\right)\left(\beta^{2}+\delta^{2}\right)}}-2 \mathrm{i} \alpha \xi\right] \operatorname{dn}\left((\gamma+\delta) W, \frac{4 \gamma \delta}{(\gamma+\delta)^{2}}\right)
$$

where

$$
W=\xi-V \tau \quad V=\frac{1}{4 \sqrt{\left(\alpha^{2}+\gamma^{2}\right)\left(\alpha^{2}+\delta^{2}\right)}} .
$$

When $\delta \rightarrow \gamma$, from (34) we obtain

$$
\begin{aligned}
Q(\tau, \xi)=4 \gamma & \exp \left(-\frac{\mathrm{i}(\alpha+\beta) \tau}{4 \sqrt{\left(\alpha^{2}+\gamma^{2}\right)\left(\beta^{2}+\gamma^{2}\right)}}-\mathrm{i}(\alpha+\beta) \xi\right) \\
& \times \operatorname{cn}\left(\sqrt{4 \gamma^{2}+(\alpha-\beta)^{2}} W, \frac{4 \gamma^{2}}{4 \gamma^{2}+(\alpha-\beta)^{2}}\right)
\end{aligned}
$$


where

$$
W=\xi-V \tau \quad V=\frac{1}{4 \sqrt{\left(\alpha^{2}+\gamma^{2}\right)\left(\beta^{2}+\gamma^{2}\right)}} .
$$

If we put $\delta=\gamma$ in (37) or $\alpha=\beta$ in (39), i.e. two pairs of $\lambda_{j}$ coalesce into one pair, we obtain the soliton solution

$$
Q(\tau, \xi)=4 \gamma \exp \left(-\frac{\mathrm{i} \alpha \tau}{2\left(\alpha^{2}+\gamma^{2}\right)}-2 \mathrm{i} \alpha \xi\right) \operatorname{sech}\left(2 \gamma \xi-\frac{\gamma \tau}{2\left(\alpha^{2}+\gamma^{2}\right)}\right)
$$

and

$$
S=-1+\frac{2 \gamma^{2}}{\alpha^{2}+\gamma^{2}} \operatorname{sech}^{2}\left(2 \gamma \xi-\frac{\gamma \tau}{2\left(\alpha^{2}+\gamma^{2}\right)}\right) .
$$

The partial case $\alpha=0$ corresponds to the real solution discussed in [1].

The other choice of sign before $a$ in (12) in this limit would give us the unstable soliton solution (see [1]).

\section{Whitham equations}

In a non-uniform case the parameters $\lambda_{i}$ become functions of the space coordinate $\xi$ and time $\tau$. The wavelength of the solution obtained in section 2 is given by

$$
L=\frac{1}{2} \oint \frac{\mathrm{d} \mu}{\sqrt{-\overline{P(\mu)}}}
$$

If this wavelength $L$ is much less than the characteristic size which the parameters $\lambda_{i}$ change considerably, then the evolution equations for $\lambda_{i}$ can be averaged over the fast oscillations with wavelength $L$, and we arrive at the Whitham equations $[4,5]$. Integrable equations possess an infinite set of conservations laws, and in this case it is convenient to average their generating function [12]. The AB system belongs to the AKNS scheme $[1,13]$ and for such equations the generating function can be taken [7-10] in the form

$$
\frac{\partial}{\partial \tau}\left(\frac{G}{g}\right)-\frac{\partial}{\partial \xi}\left(\frac{B}{g}\right)=0
$$

where the functions $f, g, h$ should be normalized according to the condition $f^{2}-g h=1$ independent of the slow change in the $\lambda_{i}$ (see [12]).

For the case of the AB system, substitution of (5), (6), (13) and (14) into (44) gives

$$
\frac{\partial}{\partial \tau}\left(\frac{\sqrt{P(\lambda)}}{\lambda-\mu}\right)-\frac{\partial}{\partial \xi}\left[\frac{\sqrt{P(\lambda)}}{4 a}\left(\frac{1}{\lambda-\mu}-\frac{1}{\lambda}\right)\right]=0 \text {. }
$$

The condition for the vanishing of the coefficients of singular terms, which result from differentiation of $\sqrt{P(\lambda)}$ with respect to $\tau$ and $\xi$, yields the equations

$$
\left\langle\frac{1}{\lambda_{i}-\mu}\right\rangle \frac{\partial \lambda_{i}}{\partial \tau}-\frac{1}{4 a}\left(\left\langle\frac{1}{\lambda_{i}-\mu}\right\rangle-\frac{1}{\lambda_{i}}\right) \frac{\partial \lambda_{i}}{\partial \xi}=0
$$

where averaging has been performed according to the rule

$$
\left\langle\frac{1}{\lambda_{i}-\mu}\right\rangle=\frac{1}{2 L} \oint \frac{\mathrm{d} \mu}{\left(\lambda_{i}-\mu\right) \sqrt{-P(\mu)}}=-2 \frac{\partial \ln L}{\partial \lambda_{i}}=2 \frac{K}{\partial_{i} K}
$$

in which $K=2 \pi / L$ is the wavevector and $\partial_{i} \equiv \partial / \partial \lambda_{i}$. Thus, we have obtained the desired Whitham equations for parameters $\lambda_{i}$ in the diagonal Riemann form

$$
\frac{\partial \lambda_{i}}{\partial \tau}+v_{i} \frac{\partial \lambda_{i}}{\partial \xi}=0 \quad i=1,2,3,4
$$


where the characteristic velocities are equal to

$v_{t}=-\frac{1}{4 a}\left(1-\frac{1}{2 \lambda_{i}} \frac{K}{\partial_{i} K}\right)=\left(1+\frac{K}{\partial_{i} K} \partial_{i}\right) \frac{1}{4 \sqrt{\prod_{k} \lambda_{k}}} \quad i=1,2,3,4$.

The form of the characteristic velocities as a result of the action of the differential operator in parenthesis on the phase velocity $V$ of the nonlinear wave expressed in terms of the Riemann invariants,

$$
v_{i}=\left(1+\frac{K}{\partial_{i} K} \partial_{i}\right) V
$$

has a general nature $[7,9,10,14,15]$ and represents, in fact, the conservation of the 'number of waves' $[4,5]$

$$
\frac{\partial K}{\partial \tau}=\frac{\partial(K V)}{\partial \xi}
$$

Here we wish to note that a slight generalization of (49) yields the most general expression for the Whitham velocities for integrable equations with the Lax pair operator $L$ corresponding to the NLS equation. Indeed, introducing the constant shift into the parameters $\lambda_{i}$

$$
\lambda_{i} \rightarrow \lambda_{i}-\Delta
$$

we obtain the phase velocity of the nonlinear periodic wave for Maxwell-Bloch equations in the sharp-line approximation [16]

$$
V=\frac{1}{4 \sqrt{\prod_{k}\left(\lambda_{t}-\Delta\right)}}
$$

Averaging of this expression over $\Delta$ with the distribution function $\tilde{g}(\Delta)$ yields the phase velocity and Whitham equations for the self-induced transparency problem with the inhomogeneously broadened line [16]

$$
V=\int \frac{\tilde{g}(\Delta) \mathrm{d} \Delta}{4 \sqrt{\prod_{k}\left(\tilde{\lambda}_{i}-\Delta\right)}}
$$

In the limit $\Delta \rightarrow \infty$, equations (50) and (53) reproduce the Whitham equations for the case of the NLS equation [17].

Let us consider this observation from a more general point of view. It is known that the Whitham equations for the NLS case

$$
\frac{\partial \lambda_{i}}{\partial \tau}+w_{i}\left(\lambda_{k}\right) \frac{\partial \lambda_{i}}{\partial \xi}=0 \quad w_{i}=\left(1+\frac{K}{\partial_{i} K} \partial_{i}\right) \sum_{k} \lambda_{k}
$$

commute with another flow with velocities (50), if $V$ satisfies the generalized Euler-PoissonDarboux equation

$$
\partial_{i j}^{2} V=\frac{1}{2\left(\lambda_{i}-\lambda_{j}\right)}\left(\partial_{i} V-\partial_{j} V\right)
$$

(see $[14,15,18,19]$ ). The system $(56)$ has the solution

$$
V=\frac{1}{\sqrt{\prod_{k}\left(\lambda_{k}-\Delta\right)}}
$$

which reproduces equation (53). Thus, all the above-mentioned cases correspond to the flows commuting with each other. Knowledge of the commuting flows allows one to construct the solutions of the Whitham equations by the generalized hodograph method $[20,21]$. However, some results can be obtained by simpler methods. One such an example is discussed in section 4. 


\section{Soliton creation on a pulse front}

Let us consider the evolution of a step-like pulse. The plane-wave solution of the AB system corresponds to $\delta=0$, and in this limit we obtain the following expression from (34) with $\beta>\alpha$ (see the analogous calculation in [6])

$$
Q(\xi, \tau)=2 \gamma \exp \left(-2 \mathrm{i} \alpha \xi-\frac{\mathrm{i} \tau}{2 \sqrt{\alpha^{2}+\gamma^{2}}}\right) \quad S=-\frac{\alpha}{\sqrt{\alpha^{2}+\gamma^{2}}} .
$$

Let the pulse have a sharp front, that is

$$
|Q(\xi, 0)|=2 \gamma \quad \text { for } \xi \leqslant 0 \quad|Q(\xi, 0)|=0 \text { for } \xi>0 .
$$

Such a front is unstable because of dispersion and modulation instability, which follow from the dispersion relation of the linear modulation wave of the plane-wave solution (58):

$$
\Omega=\frac{1}{2 \sqrt{\alpha^{2}+\gamma^{2}}} \cdot \frac{K \sqrt{K^{2}-4 \gamma^{2}}-2 \alpha K}{K^{2}-4\left(\alpha^{2}+\gamma^{2}\right)} .
$$

This dispersion relation was obtained by linearization of the $A B$ system with respect to a small periodic perturbation of (58) with wavevector $K$ and frequency $\Omega$. We see that the modulation waves with $K \geqslant 2 \gamma$ are stable, but for $K<2 \gamma$ there is modulation instability. Due to dispersion and this instability, the localized disturbance (such as a sharp front in our case) will change its form at the linear stage, and one can expect that after some time it will transform into the non-uniform expanding region which can be described as a modulated nonlinear periodic wave in which the parameters $\alpha, \beta, \gamma, \delta$ are slow functions of $\xi$ and $\tau$, so that we can apply the Whitham method. Gurevich and Pitaevskii [11] were the first to apply such an approach to this kind of problems. In the case of unstable systems it was used for the first time in $[3,7]$. We shall follow here the methods of those articles.

In the problem under consideration there is no characteristic dimension, hence the parameters $\lambda_{i}$ depend only on the self-similar variable $\zeta=\xi / \tau$. Since $\lambda_{3}=\lambda_{1}^{*}$ and $\lambda_{4}=\lambda_{2}^{*}$, it is sufficient to use only two Whitham equations (48), which in our self-similar case take the form

$$
\frac{\mathrm{d} \lambda_{1}}{\mathrm{~d} \zeta}\left(v_{1}-\zeta\right)=0 \quad \frac{\mathrm{d} \lambda_{2}}{\mathrm{~d} \zeta}\left(v_{2}-\zeta\right)=0
$$

As we shall see, the solution corresponding to our initial data (59) is $\lambda_{1}=$ constant, $v_{2}=\zeta=\xi / \tau$ or

$$
\begin{gathered}
\alpha+\mathrm{i} \gamma=\text { constant } \\
\frac{1}{4 \sqrt{\left(\alpha^{2}+\gamma^{2}\right)\left(\beta^{2}+\delta^{2}\right)}}\left\{1-\frac{1}{\beta+\mathrm{i} \delta} \frac{2 \mathrm{i} \delta[\alpha-\beta+\mathrm{i}(\gamma-\delta)] \mathrm{K}(m)}{[\alpha-\beta+\mathrm{i}(\gamma-\delta)] \mathrm{K}(m)-[\alpha-\beta+\mathrm{i}(\gamma+\delta)] \mathrm{E}(m)}\right\} \\
=\frac{\xi}{\tau}
\end{gathered}
$$

where $\mathrm{K}(m)$ and $\mathrm{E}(m)$ are the complete elliptic integrals. On separating real and imaginary parts in the above equations, we obtain the equations

$$
\begin{aligned}
& \frac{E(m)}{\mathrm{K}(m)}=\frac{\beta\left(\alpha^{2}+\beta^{2}+\gamma^{2}+\delta^{2}\right)-2 \beta(\alpha \beta+\gamma \delta)}{\beta\left(\alpha^{2}+\beta^{2}+\gamma^{2}+\delta^{2}\right)-2 \alpha\left(\beta^{2}+\delta^{2}\right)} \\
& \quad-\frac{1}{4 \sqrt{\left(\alpha^{2}+\gamma^{2}\right)\left(\beta^{2}+\delta^{2}\right)}} \cdot \frac{\alpha\left(\beta^{2}+\delta^{2}\right)-\beta\left(\alpha^{2}+\gamma^{2}\right)}{(\alpha-\beta)\left(\beta^{2}+\delta^{2}\right)}=\frac{\xi}{\tau}
\end{aligned}
$$


which, together with $\alpha=$ constant, $\gamma=$ constant and equation (30), determine implicitly the dependence of $\beta$ and $\delta$ on $\zeta=\xi / \tau$.

The end-points of non-uniform oscillating region correspond to the limits $m \rightarrow 0$ and $m \rightarrow 1$. For investigation of these limits it is convenient to express $\beta$ and $\delta$ as functions of $m$ (as was done in [3] in the case ot the NLS equation). The solution of (30), (63) and (64) has the form

$$
\begin{gathered}
\beta=\frac{\alpha}{\alpha^{2}+\gamma^{2} m^{2} A^{2}(m)} \cdot\left(\alpha^{2}-\gamma^{2} m A(m)+2 \alpha \gamma^{2} A(m)\right. \\
\left.+\sqrt{4 \alpha^{2} A(m)+4 \gamma^{2} A^{2}(m)(1-m)-(1+m A(m))^{2}}\right) \\
\delta=\frac{\gamma}{\alpha} m A(m) \beta
\end{gathered}
$$

where we have introduced the function

$$
A(m)=\frac{(2-m) \mathrm{E}(m)-2(1-m) \mathrm{K}(m)}{m^{2} \mathrm{E}(m)} .
$$

If $m \rightarrow 1$ we have

$$
\beta \simeq \alpha\left(1+\frac{2 \gamma \sqrt{1-m}}{\sqrt{\alpha^{2}+\gamma^{2}}}\right) \quad \delta \simeq \gamma\left(1+\frac{2 \gamma \sqrt{1-m}}{\sqrt{\alpha^{2}+\gamma^{2}}}\right)
$$

and according to (64) this edge point moves with the soliton velocity

$$
v_{\mathrm{s}}=\left.\frac{\xi}{\tau}\right|_{m \rightarrow 1}=\frac{1}{4\left(\alpha^{2}+\gamma^{2}\right)} \text {. }
$$

If $m \rightarrow 0$, then $\beta$ and $\delta$ tend to the limiting values

$$
\beta=\alpha\left[1+\frac{3 \gamma^{2}}{4 \alpha^{2}}\left(1+\sqrt{1+\frac{8 \alpha^{2}}{9 \gamma^{2}}}\right)\right] \quad \delta=0
$$

and equation (64) takes the form

$$
v=\frac{\xi}{\tau}=\frac{\alpha^{2}+\gamma^{2}-\alpha \beta}{4 \beta^{2}(\alpha-\beta) \sqrt{\alpha^{2}+\gamma^{2}}} .
$$

In this limit of small modulation the Whitham theory must reproduce the linear theory, that is $v$ must coincide with the corresponding group velocity of the modulation wave. At small $\delta$ equation (29) gives:

$|Q|^{2} \simeq 4 \gamma^{2}+4 \gamma \delta\left[\exp \left(\mathrm{i} 2 \sqrt{(\alpha-\beta)^{2}+\gamma^{2}}\left(\xi-\frac{\tau}{4 \beta \sqrt{\alpha^{2}+\gamma^{2}}}\right)\right)+\mathrm{CC}\right]$

that is

$$
K=2 \sqrt{(\alpha-\beta)^{2}+\gamma^{2}} \quad \Omega=\frac{K}{4 \beta \sqrt{\alpha^{2}+\gamma^{2}}} .
$$

It is easy to check that these values of $K$ and $\Omega$ satisfy the dispersion relation (60). Calculation of group velocity $v_{\mathrm{g}}=\mathrm{d} \Omega / \mathrm{d} K$ at this value of $K$ reproduces, as we expected, equation (71). One can find that $v_{\mathrm{g}}<v_{\mathrm{s}}$ for all $\alpha$ and $\gamma$, if $\beta$ is given by (70).

We see that the sharp front transforms into the expanding oscillatory region. The faster edge of this region moves with the soliton velocity and consists of the train of solitons. The slower edge propagates with the group velocity of the small modulation wave. The whole region can be described as a modulated nonlinear periodic solution of the AB system. 
The emerging picture looks Jike the so-called 'nondissipative shock wave' [11,22, 23], but in our case the system of Whitham equations is not hyperbolic, but elliptic. The solution found is stable at both its edges-in the soliton region and in the small modulation region (where $K>2 \gamma$, as one can see from (72)). This example and the results presented in $[3,7]$ show that Whitham theory is rather effective in such unstable (elliptic) cases, too.

\section{Conclusion}

Examples of derivation of periodic solutions and the corresponding Whitham equations by the methods suggested and applied in [6-10] show that these methods are rather convenient and useful for all integrable equations described by the AKNS scheme. By these methods we obtain the periodic solution in an effective form, and the form of the corresponding Whitham equations reflects their symmetry properties appearing due to the existence of commuting flows. Knowledge of the periodic solutions and Whitham equations permits one to investigate concrete physical problems, as was illustrated in section 4.

\section{Acknowledgments}

We are grateful to G A El', A V Gurevich and A L Krylov for stimulating discussions. MVP is grateful to the International Science Foundation for partial financial support of this work through ISF grant RKR000.

\section{References}

[1] Dadd R K, Eilbeck J C, Gíbbon J D and Morris H C 1982 Solitons and Nonlinear Wave Equations (New York: Academic)

[2] Gibbon J D and McGuiness M J 1981 Proc. R. Soc. A 337165

[3] El' G A, Gurevich A V, KhodorovskiY V V and Krylov A L 1993 Phys. Lett. 177A 357

[4] Whitham G B 1965 Proc. R. Soc. A 283238

[5] Whitham G B 1974 Linear and Nonlinear Waves (New York: Wiley)

[6] Kamchatnov A M 1990 J. Phys. A: Math. Gen. 232945

[7] Kamchatnov A. M 1992 Phys. Lett. 162A 389

[8] Kamchutnov A M 1992 Zh Exp. Teor. Fiz, 1021606 (Engl. transi. Sov. Phys.-JETP 75 868)

[9] Kamchatnov A M 1990 Zh. Exp. Teor. Fiz 97144 (Engl. transl. Sov. Phys.-JETP 70 80)

[10] Kamchatnov A M 1994 Phys. Lett. 186A 387

[11] Gurevich A V and Pitaevskii L P 1973 Zh. Exp. Teor. Fiz, 65590 (Engl, transl. Sov. Phys.-JETP 38 291)

[12] Flaschka H, Forest M G and McLaughlin D W 1980 Commun. Pure Appl. Math. 33739

[13] Ablowitz M J, Kaup D J, Newell A C and Segur H 1974 Stud. Appl. Math. 53241

[14] Gurevich A V, Krylov A L and El' G A 1991 Pis'ma Zh. Exp. Teor. Fis. 54104 (Engl. transl. JETP Lett. 54 102)

[15] Kudashev V R 1991 Pis'ma Zh. Exp. Teor. Fiz. 54179

[16] Kamchatnov A. M and Pavlov M V 1995 Zh. Exp. Teor. Fiz. 10744

[17] Pavlov M V 1987 Teor. Mar. Fiz, 71351

[18] Kudashev V R and Sharapov S E 1991 Teor. Mat. Fiz 8740

[19] Pavlov M V 1992 Thesis Landau Institute

[20] Tsarev S P $1990 \mathrm{lzv}$. Akad. Nauk. Ser. Mat 541048

[21] Dubrovin B A and Novikov S P 1989 Usp. Mat. Nauk 4429

[22] Gurevich A V and Krylov A L 1987 Zh. Exp. Teor. Fiz. 921684 (Engl. transl. Sov. Phys.-JETP 65 944)

[23] Gurevich A V, Kryloy A L and El' G A 1992 Zh. Exp. Teor. Fiz 1021524 (Engl, transl. Sov, Phys.-JETP 75 825) 